Ten patients (none with cenocepacia) are using inhaled antibiotics (3 colistin, 1 Colobreath, 2 TOBI, 2 Cayston, 1 ceftazidime, 1 alternating Cayston and Promixin).

Conclusion This study shows that a significant proportion of our Burkholderia spp infected patients have organisms that are sensitive to currently available inhaled antibiotics. Given our positive experience, and with the expected availability of new inhaled antibiotics in the near future, perhaps the time has come to formally look at the use of inhaled anti-microbial therapy in this small but important cohort of CF patients.

\section{P230 INVESTIGATING THE ROLE OF CHEST PHYSIOTHERAPY IN THE COLLECTION OF SPUTUM SAMPLES FROM INDIVIDUALS WITH CYSTIC FIBROSIS (CF)}

R Dacie, R Howlin, M Carroll, G Connett. University of Southampton, Southampton, UK

\subsection{6/thoraxjnl-2015-207770.366}

Introduction Chronic respiratory infection is responsible for the majority of the morbidity and mortality of CF patients. In order to guide treatment regimes and improve understanding of the pathophysiology of CF, airway secretions are sampled and analysed. Sputum is usually the selected method of sampling.

Often, large quantities of sputum are required to facilitate comprehensive laboratory testing. Hence, when designing studies, it is important to consider the quantity of sputum likely to be produced by patients and to ensure that the composition of the sputum samples is not altered by the procedure by which they are obtained.

This study aimed to investigate the effect of chest physiotherapy on the quantity and composition of sputum samples collected from individuals with CF. It was hypothesised that physiotherapy would increase the quantity of sputum produced, reduce the salivary content and alter the microbiological content. Methods Clinically stable adults with CF were recruited at outpatient clinics and randomised into group A (physiotherapy group, $\mathrm{n}=21$ ) or group B (no physiotherapy group, $\mathrm{n}=25$ ). Laboratory processing of the samples involved determining sample weights and counting human cells (alive respiratory cells, dead respiratory cells and squamous cells). The dissolved sputum was also transferred onto plates of cetrimide agar for culturing Pseudomonas aeruginosa. Colony-forming units (CFUs) were counted on the plates after $24 \mathrm{~h}$.

Results Samples from the physiotherapy group had significantly greater weights than the no physiotherapy group $(\mathrm{p}<0.001)$. When considering the total number of cells per gram of sputum, there was no statistical difference between the two groups ( $\mathrm{p}=$ 0.396). However, the numbers of squamous cells per gram, and dead respiratory cells per gram were both significantly greater in the no physiotherapy group $(\mathrm{p}=0.039$ and $\mathrm{p}=0.001$ respectively). There were no significant differences between numbers of alive respiratory cells per gram $(\mathrm{p}=0.487)$ or CFUs $(\mathrm{p}=$ $0.459)$.

Conclusion Whilst physiotherapy was found to increase the quantity of sputum collected, there were significant differences in sputum composition, suggesting that the two groups represent samples from different niches. Hence, when planning a study involving sputum analysis, the procedure by which the sample is obtained has to be considered when interpreting the results.

\section{P231 A PROSPECTIVE COHORT STUDY OF INTEGRATED PALLIATIVE CARE OF CYSTIC FIBROSIS (CF)}

SJ Bourke, R Mackley, Z Booth, S Doe, A Anderson, S Rice, AD Gascoigne, R Quibell. Royal Victoria Infirmary, Newcastle Upon Tyne, UK

\subsection{6/thoraxjnl-2015-207770.367}

There are 140 deaths in the UK each year from CF, often on a transplant waiting list and often without specialist palliative care. A palliative physician and nurse joined our team in 2011, providing palliative care in parallel with standard CF care. We undertook a prospective study documenting symptoms and outcomes, the views of the CF team and the experience of the palliative specialists.

Over 3 years, $28(10 \%)$ of 282 patients at our Centre had palliative input; their mean age was 31 (range 18-47) years and mean FEV1 was $0.86 \mathrm{~L}(24 \%) ; 17(61 \%)$ died - 6 were on a transplant waiting list, 10 were unsuitable, and one died post transplantation; 4 have had transplantation and no longer need palliative input, 7 are in on-going care; 15 (88\%) of deaths were on the CF ward and 2 at home. All patients who died had had palliative care. The main symptoms were breathlessness, cough, pain, vomiting, fatigue and low mood. The mean palliative assessment score was high at 2.9, indicating that life was dominated by symptoms. Palliative interventions included opioid, benzodiazepine, anti-emetic and anti-depressant medications and non-pharmacological interventions included relaxation techniques, massage, acupuncture and cognitive therapy. A survey was completed by 16 members of the CF team: all felt that palliative specialists should be part of the team and rated the model of care highly with a mean score of 4.1 (scale 1-5); 11 thought that patients had found input very helpful and 5 helpful; one patient declined a palliative consultation. The palliative specialists had increased their knowledge of CF, found it useful to meet patients earlier and had no difficulty in providing palliation in parallel with standard CF care. Their workload was high and they identified additional needs of bereavement counselling and managing the effects of deaths on other CF patients.

This integrated model was successful in overcoming barriers to specialist palliative care. Palliative specialists have improved their knowledge of $\mathrm{CF}$ and the $\mathrm{CF}$ team have learnt palliative skills.

\section{P232 TOO SWEET FOR TOO LONG?}

S Ali, R Khetan, P Sachdev, J Bhatt. Nottingham Univeristy Hospitals NHS Trust, Nottingham, England, UK

\subsection{6/thoraxjnl-2015-207770.368}

Background Cystic fibrosis related diabetes (CFRD) is associated with deterioration in clinical status. Lung function and nutritional status deteriorate up to 2-4 years before a diagnosis of CFRD based on the oral glucose tolerance test (OGTT). Timely detection and treatment is crucial.

Aims To evaluate:

- adherence to CFRD screening guidelines and

- whether identifying stages of progressive Cystic Fibrosis Insulin Deficiency (CFID) using the extended OGTT altered management

- trends in weight, BMI and FEV1 in CFRD as compared to CF controls. 